\title{
An overview of computational photography
}

\author{
SUO JinLi, JI XiangYang \& DAI QiongHai* \\ Department of Automation, Tsinghua University, Beijing 100084, China
}

Received November 2, 2010; accepted November 26, 2011

\begin{abstract}
Computational photography is an emerging multidisciplinary field. Over the last two decades, it has integrated studies across computer vision, computer graphics, signal processing, applied optics and related disciplines. Researchers are exploring new ways to break through the limitations of traditional digital imaging for the benefit of photographers, vision and graphics researchers, and image processing programmers. Thanks to much effort in various associated fields, the large variety of issues related to these new methods of photography are described and discussed extensively in this paper. To give the reader the full picture of the voluminous literature related to computational photography, this paper briefly reviews the wide range of topics in this new field, covering a number of different aspects, including: (i) the various elements of computational imaging systems and new sampling and reconstruction mechanisms; (ii) the different image properties which benefit from computational photography, e.g. depth of field, dynamic range; and (iii) the sampling subspaces of visual scenes in the real world. Based on this systematic review of the previous and ongoing work in this field, we also discuss some open issues and potential new directions in computational photography. This paper aims to help the reader get to know this new field, including its history, ultimate goals, hot topics, research methodologies, and future directions, and thus build a foundation for further research and related developments.
\end{abstract}

Keywords computational photography, computational imaging, plenoptic function, light field

Citation Suo J L, Ji X Y, Dai Q H. An overview of computational photography. Sci China Inf Sci, 2012, 55: 1229-1248, doi: 10.1007/s11432-012-4587-6

\section{Introduction}

From film photography to digital photography, the simplicity of capture and the display quality of visual information have both greatly improved. By combining the strong image capture capability of digital cameras with the development of computational technology, researchers can perform a series of processes on the captured digital images, including image processing, image synthesis, and image understanding. However, traditional photography and the related processing are usually limited to the existing imaging principles, imaging equipment manufacturing technology and imaging environments, and thus are far from being sufficient to satisfy the growing demand.

*Corresponding author (email: qionghaidai@tsinghua.ed.cn) 


\subsection{What is computational photography?}

Computational photography emerged in the 1990s and has developed rapidly over the last two decades. As yet, there is neither a clear boundary nor a concrete definition of computational photography. As a loose description, computational photography accounts for studies for the extension of film-like photography and digital photography, which both capture only a $2 \mathrm{D}$ projection of visual scenes. The difference is that computational photography aims to acquire much richer visual information, to generate images with compelling visual results or assist with specific tasks, e.g. computer vision or medical science.

Computational photography is a highly multidisciplinary field, which is related closely to research in computer vision, computer graphics, signal processing, materials, hardware design, and similar disciplines, and attracts interest from researchers, artists, photographers, and engineers. Thanks to the efforts of the large numbers of people involved in this new area, major progress has been made in this field.

\subsection{The goals of computational photography}

To capture visual information in more effective and empowering ways, computational photography considers every step of the imaging process. These related studies in computational photography stem from the following objectives:

1) To break the limitations of traditional photography, to better acquire and display real visual scenes;

2) To help to advance the various fields concentrated on digital imaging, e.g. computer vision, computer graphics;

3) To meet the increasing demands of real-life imaging applications, such as astronomical photography, medical imaging, and surveillance.

Because computational photography is an emerging field, the goals of computational photography are not limited to the aforementioned items, and will certainly be extended with increasing real-life demands and advances in related fields.

\subsection{History of computational photography}

The pursuit of good photographs started as early as the invention of the film camera, and is attracting increasing attention because of the availability of digital cameras (especially the single-lens reflex camera), which provide higher image resolution, more convenient image capture and greater space for the postprocessing of photographs. Based on the strong capabilities of digital imaging, researchers continue to conduct a great deal of image analysis to further advance the progress made in computer vision, computer graphics, and signal processing (particularly image processing), and subsequently to promote the development of imaging in suitable application fields such as medical fields, remote sensing and entertainment.

Despite the rapid development of the technology, the digital camera is still unable to satisfy all of the needs of photographers, and photograph-based tasks are also limited to a large extent. One of the most important theoretical causes of these limitations is that the traditional photography mechanism involves the integration of light from an object into two dimensions, and it is quite different from the visual signal in real world.

1) From traditional photography to computational photography. To extract the visual information from the real world completely, Adelson et al. [1] proposed the plenoptic function in 1991 to describe the light rays in the real world. Later, in [2], they implemented a prototype for the capture of a 4D subspace of the plenoptic function, named the plenoptic camera. After capturing the whole plenoptic function, one can reconstruct the information for any object, from at any viewpoint, at any time constant, and within any wavelength range.

Inspired by the plenoptic function and motivated by the limitations of traditional photography, researchers from computer vision, computer graphics, signal processing, materials and other fields began research towards a breakthrough. Two short courses were held at Stanford in 2004 and at MIT in 2005 
separately, from which the term "computational photography" was proposed formally. The word "computational" means to endow the digital camera with computational capability to some extent, to extend the capabilities of traditional photography.

2) Increasing research interest and fruitful studies. Since computational photography was proposed, major efforts have gone into this emerging field. In addition to the researchers in closely related fields (e.g. computer vision, computer graphics, signal processing and applied optics.), photographers, camera manufacturers and hardware engineers were also involved.

Despite its short history, computational photography has developed rapidly and has become a hot research field in academia. Numerous universities and research institutes, including Stanford University, Massachusetts Institute of Technology, Carnegie Mellon University, New York University, Columbia University, and Max-Planck-Institut für Informatik, have opened related courses, and many international conferences in both computer vision and computer graphics have set special sessions for computational photography. Also, the 1st, 2nd and 3rd International Conferences on Computational Photography (ICCP) have been held in 2009, 2010 and 2011, respectively.

In summary, this wide level of exploration has brought prosperous development to this relatively new field, and computational photography has become a hot topic in academia.

\section{Elements of a computational imaging system}

The photographic process is composed of two stages: in the sampling stage, light emitted from light sources is transported through a series of optical elements and integrated at the image sensor; in the reconstruction stage, the signals from the sensor are transformed into a digital image for final visualization, and the reconstruction should be coupled with the preceding computational sampling process. However, these two stages in computational photography are different from those in traditional digital/film imaging, because some computations are necessary in both stages, and thus they are called "computational" sampling and reconstruction (as shown in Figure 1).

\subsection{Computational sampling}

During the sampling stage, every element of a digital photograph can be improved for better visual information capture, and so the related topics in computer photography cover every imaging step (as shown in the left column of Figure 1), including the optics, sensors, and external illumination.

1) Computational optics. Most typical computational optics try to improve the imaging results by controlling the aperture and capturing optically coded images, i.e., coded aperture imaging. In previous work, various finely designed modulators have been inserted into the aperture or added in front of the camera for the capture of optically coded images, including masks[3-7], pinhole patterns [8], lenslets [911], filters or occluders [12-15], and mirrors [16-20]; these elements are used in specific applications, such as capture of the light field [21], long distance camera interaction [22], defocus deblurring [23], extending the depth of field or refocusing [4-7,23-25], extending the field of view [26,27], confocal imaging [20], enhancing the dynamic range $[13,18,28,29]$, extracting the depth cues [15], and capturing multi-modal visual information [30].

Instead of introducing external elements, some researchers modify the optics in other ways. For example, Zomet et al. [26] used a set of parallel light attenuating layers to replace a lens, Levin et al. [24] designed a lattice focal lens, and Raskar et al. [31] coded the aperture by controlling the on/off state of the shutter.

2) Computational sensor. The computational sensor attempts to design or modify the detectors in some way to obtain task-specific imaging results. Similar to the computational optics, various plug-in elements have been designed, including lens arrays [2,32], masks [6,33,34], filter arrays [35-37], and mirrors [38], for task specific imaging results, e.g. capturing the light field [2,6,32], removing veiling glare [34,39], extending the dynamic range [35-37], and increasing the field of view [38,40]. 


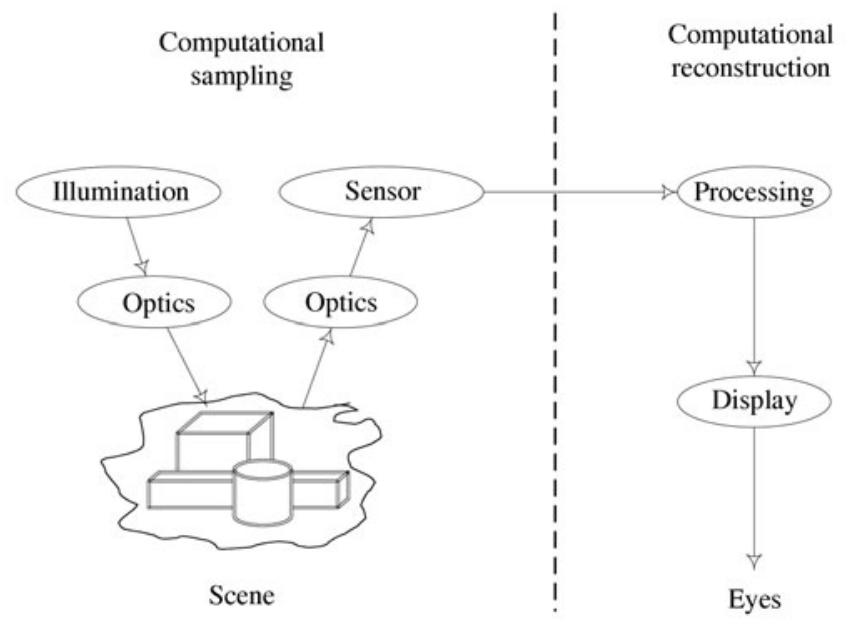

Figure 1 The elements of a computational photography system.

Some other implementations of computational sensors include the introduction of sensor motion to extend the depth of field [41,42] or perform motion deblurring [43], and building sensing patterns for image super resolution [44] or high dynamic range imaging [45,46]

3) Computational illumination. Computational illumination usually controls the photographic illumination in a structured way, to create new images that meet specific demands by introducing some computational strategies.

The intended applications can cover increasing the depth of field [47], separation of the illuminations, scene synthesis [48], relighting [49-51], adaptive color primaries, metamer detection, scene contrast enhancement, photography of fluorescent objects, high dynamic range photography [22], removing disturbances or dazzling [19], assisting with segmentation [49], image denoising or artifacts removal $[10,52,53]$, image processing or editing (e.g. detail transfer, white-balancing, continuous flash and red-eye removal) [52,54,22], specular artifacts removal [55], estimation of the ambient illumination [56], depth estimation [57-60], light field transfer [48], shadow detection [61], and extracting middle-level features (e.g. shape, surface, and reflectance) [14].

For illumination encoding, the various design schemes can be divided into the following subclasses:

(i) The design of coded lighting patterns, e.g. use of a periodic stripe pattern [62], a dense binary illumination pattern [63,64], a programmable lighting dome [50,51,65-67], light positioning from near to far [68], a light source array arranged along specific paths [66,69], a lighting design [70], or a light placed behind specific occluders [71].

(ii) Collection of images under different spectra by using multiple light sources, applying diffraction grating or conducting program control $[19,22,49]$, etc.

(iii) Capture of multi-flash images [57-60,55] or flash/no-flash image pairs [10,70,56,54,52].

\subsection{Computational reconstruction}

As mentioned earlier, specific computations are necessary to transform the sensed signals into the 'final' images during the computational reconstruction stage (see the right column of Figure 1). Similar to other general signal processing tasks, the formation of digital images can be analyzed in both the spatial and frequency domains using basic signal processing theory.

In the considerable literature of computational photography research, most studies attempt to model or modify the light ray transport in the spatial domain, while some researchers study the light field in the Fourier domain. Representative work includes: Veeraraghavan et al. [6,72], who designed mask enhanced cameras to heterodyne the band-limited light fields in the frequency domain for refocusing; $\mathrm{Ng}$ [32], who analyzed the 4D light field captured by a lenslet based camera [12] in the Fourier domain and selected its specific slice trajectory for refocusing; and Georgiev et al. [73], who analyzed the light field cameras under a unified framework in the frequency domain. Other researchers performed demosaicing [74] or computed 
3D occluders and cast shadows [75] in the frequency domain. Despite the large differences between the analyses in the two domains, Ihrke et al. [76] proved that the spatial techniques in computational photography can find a corresponding explanation in the frequency domain, and vice versa.

As noted above, computational photography provides advantages over previous work in some aspects by performing modifications to the imaging elements, and various prototypes have been proposed by the various researchers. To build a portable and unified platform for research in this field, Adams et al. [77] proposed an imaging architecture named Frankencamera and developed two implementations.

\section{Image properties benefited from computational photography}

Because of the intrinsic limitations of traditional imaging principles and camera designs, the captured images often fail to achieve the expected visual properties, e.g. field of view, dynamic range, depth of field, wavelength/spatial/temporal resolution, as illustrated in Figure 2. By modifying the elements of the digital cameras as described in Section 2, computational photography aims to extend one or more of these limitations. In this section, we review the related studies that benefit these parameters. Because of page limitations, we only give several examples here and refer the readers to the references for more details.

\section{$3.1 \quad$ Field of view}

In photography, the field of view is the part of the world that is visible through the camera at a particular position and orientation in space. Researchers have adopted various methods for field-of-view extension:

(i) Modification or addition of camera elements, e.g. Kuthirummal et al. [38] and Taguchi et al. [78] introduced flexible mirrors, while Nomura et al. [40] arranged sensors flexibly.

(ii) Stitching of images snapped from different viewpoints, which can either be captured sequentially [20,79-83] via a traditional digital camera or captured simultaneously via a camera array [84,85], i.e., in a panorama.

\subsection{Dynamic range}

Photographers use 'dynamic range' for the luminance range of a scene being photographed, or the limits of luminance range that a given digital camera or film can capture. To increase the dynamic range of captured images, many algorithms have been proposed over the last few years.

(i) Stitching of multiple images at different exposure settings is the most intuitive approach, and plenty of algorithms $[11,86,87]$ have been developed to selectively combine multiple exposures of the same scene to increase the dynamic range. There are two main schemes to acquire multiple exposure images: The first is to acquire multiple exposure images sequentially, as used in [88-92]. However, this type of approach is only suited to stationary scenes and leads to two problems, i.e., misaligned photographs and blurred long exposure photographs [93]. The second method is to acquire multiple exposure images simultaneously via a camera with multiple sensors [94,95], a set of cameras [96], or a special sensor including multiple sensing elements with different light sensitivities [97-99].

(ii) Performing post processing by introducing various filters, such as in [98], or by performing interpolation under prior structural constraints $[17,100]$.

(iii) Modifying the sensor responses, e.g. Mitsunaga et al. [101] performed high dynamic range imaging by estimating the sensor response first and performing specific modifications, while Tumblin et al. [45] designed a sensor recording image gradient, and Wetzstein et al. [46] inserted plug-in filters (e.g. graduated neutral density filters) in front of the lens or sensor.

(iv) Designing new sensors, although this is mostly relevant to the hardware design [102-109]. 


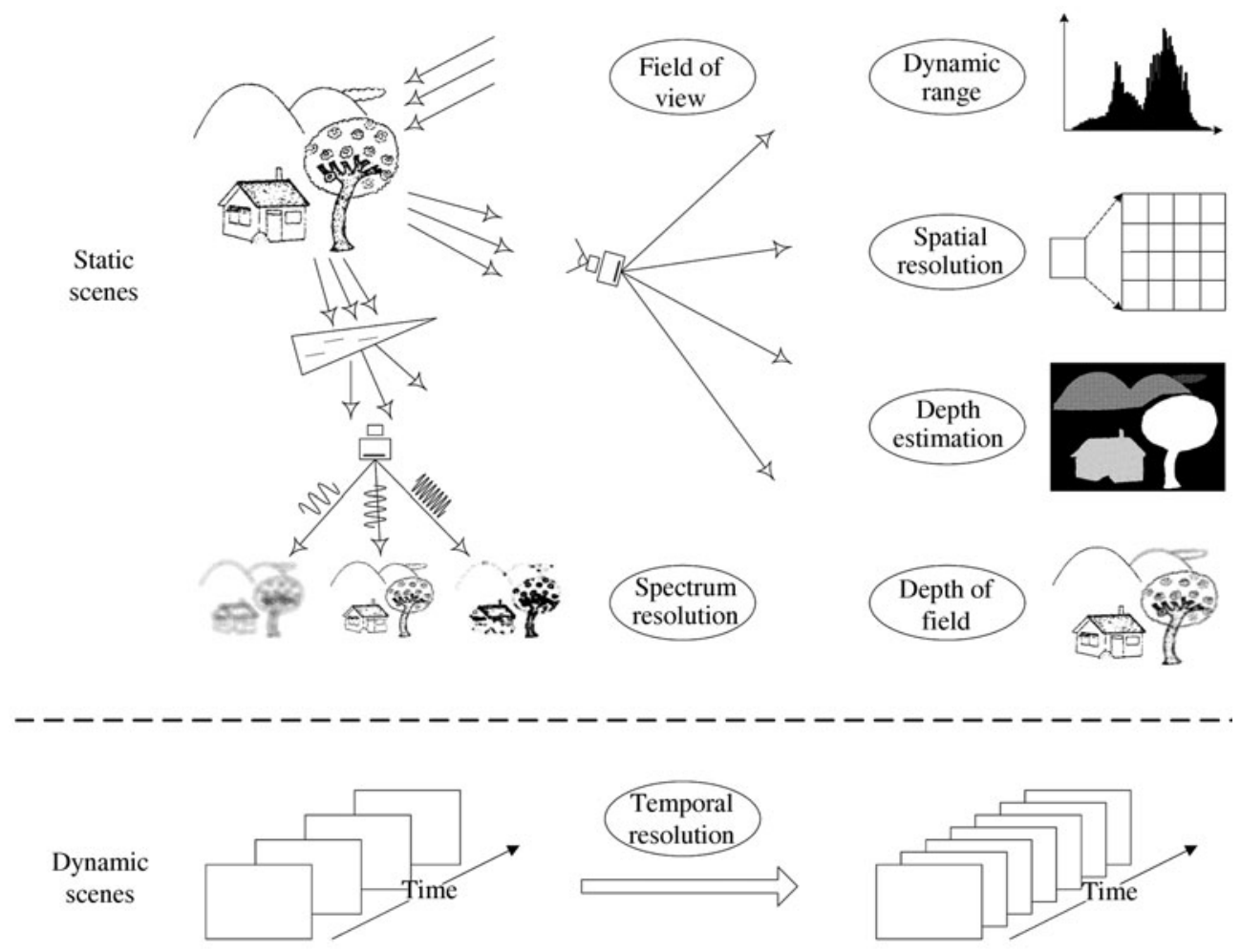

Figure 2 Different applications benefiting from the developments of computational photography.

\subsection{Depth of field}

The depth of field is the portion of a scene that appears with acceptable sharpness in the image. In traditional photography, the depth of field is usually limited to a small range, and the computational enhancement approaches fall into following categories:

1) Introduction of motion to the sensors or optics, meaning that one can perturb the lens and sensors [41] or only the sensors [42] along the optical axis during image integration to generate a depthindependent defocus kernel, to then perform spatially varying optical deblurring, i.e., extending the depth of field.

2) Generalized illumination, where, to enhance the external illumination, some researchers have projected a stripe pattern for depth estimation and performed defocus blur compensation [62].

3) Coded exposure, where, by making use of the close relationship between the aperture and the depth of field, researchers proposed two coded exposure strategies for extending the depth of field: (i) attaching a lattice-focal lens [24], a diffuser [110], or a phase mask [5] to generate a point spread function and an optical transfer function that is invariant to optical defocusing; (ii) capturing multiple images at different exposure settings $[111,112]$ instead of a single shot image, and combining them for an all-in-focus image. Hasinoff et al. [113] also studied the most efficient capture mode within a fixed time budget.

4) Multi-spectrum imaging, where, by considering the fact that different light spectra have different depths of field, Guichard et al. [114] copied the high frequencies of the sharpest color onto the other colors and obtained an increased depth of field.

5) A synthetic aperture, where light field cameras can capture images at different depths, and then integrate the refocused image with a desired virtual aperture; such approaches are presented in $[9,84,12,32,115]$.

\subsection{Wavelength resolution}

Light has different properties at different wavelengths, and one can take advantage of the different spectra in many applications [28], e.g. including material or object recognition, color analysis and color constancy, 


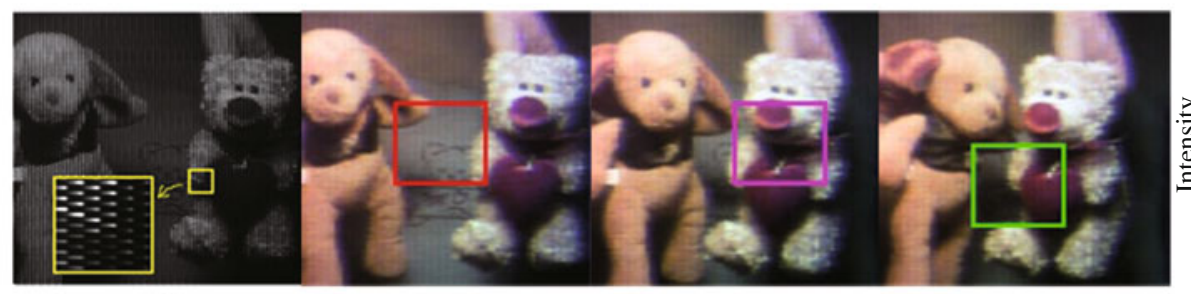

(a) (b) (c)

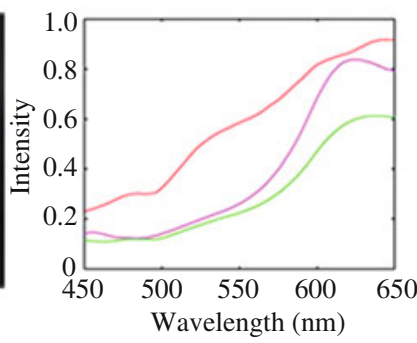

(e)

Figure 3 Results generated by approach proposed by Cao et al. [118] with high spectrum resolution and spatial resolution. (a) shows a rectified multispectral video frame captured under tungsten illumination; (b)-(d) are the resulting RGB video frames generated from (a); (e) shows the average spectra of pixels within the rectangles marked in (b)-(d).

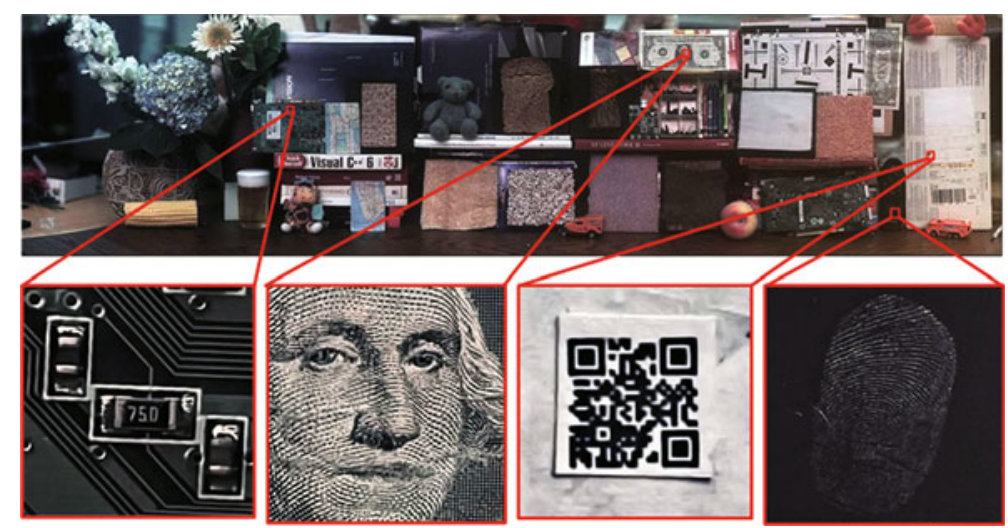

Figure 4 An example of giga-pixel image captured by [123]. The image dimensions are $82000 \times 20000$ pixel and the scene occupies a $126 \times 32^{\circ} \mathrm{FOV}$.

biomedical imaging, remote sensing and astronomy. The adopted strategies for image capture with different spectra include: introducing a filter array [114,116] or prism splitter [117], performing mechanical or electronic control [22], and computational synchronization [36]. It is possible to capture dynamic scenes with high spectrum resolution and spatial resolution simultaneously, as demonstrated by Cao et al. [118], as shown in Figure 3.

\subsection{Spatial resolution}

Limited resolution can be attributed to many possible sources, e.g., blur caused by motion or camera shake, defocus blur, limited sensor size. In this subsection, we refer to the last type only.

High resolution images can be obtained via professional cameras, but these cameras are usually costly. The spatial resolution can be enhanced computationally: e.g., Wilburn et al. [84] captured high resolution images using a large camera array, while Bishop et al. [119] raised image resolution by introducing prior constraints, and Landolt et al. [120] attained the same goal by introducing mechanical vibrations (named jittering) to the image sensor; Wang et al. [121] and Ben-Ezra [122] designed large format cameras for high resolution image capture. One newly proposed work is the giga-pixel imaging proposed by Cossairt et al. [123], who designed a compact architecture composed of a ball lens shared by several small planar sensors, and a post-capture image processing stage; one example image captured by their prototypes is shown in Figure 4.

\subsection{Temporal resolution}

Cameras shake or object motion during the exposure time leads to objectionable image blurring, and to capture the deblurred images or videos via a commercially available camera is a challenging task. One 


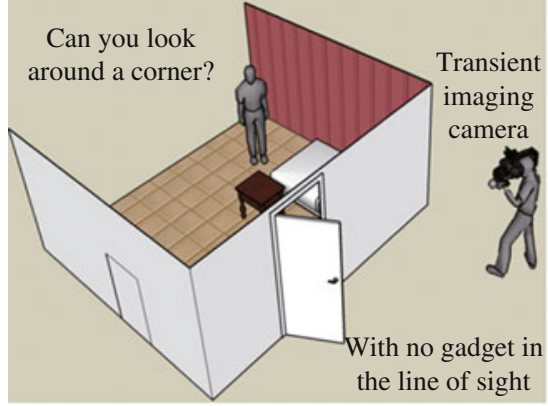

(a)

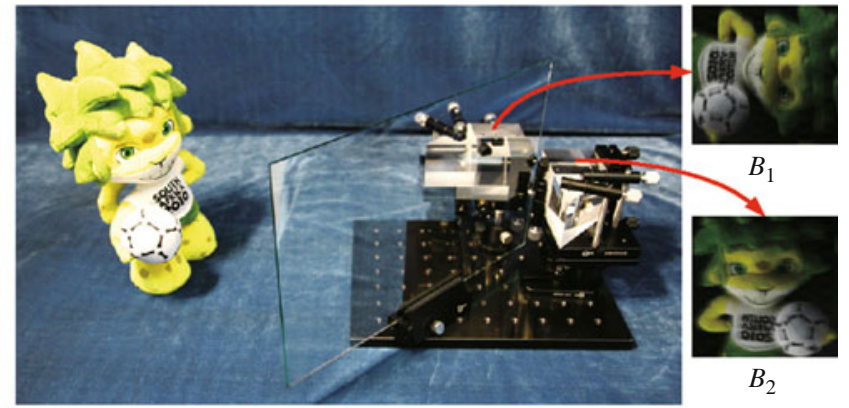

(b)

Figure 5 Two systems capturing visual information at high temporal resolution. (a) illustrates the femto system proposed by [124] and (b) gives the systems proposed by [21] for camera shake removal.

way to capture these high speed dynamics is to raise the frame rate via new camera elements; a representative work on the capture of super-high speed dynamics was proposed by Kirmani et al. [124], who used a femtosecond laser and an ultrafast photodetector array to record the light path and infer the scene structure, as illustrated in Figure 5(a). Another way is to encode the high frequency information of the moving objects or the relative motion between the camera and the scene in some sense, and perform better restoration. The various approaches have been proposed, including: interleaving a camera array in chronological order [84,125], using a hybrid array [126]; coded photography (e.g., coded exposure [31,127-131], coded sampling [132,133,21](see Figure 5(b)), coded sensor motion [134,135]); temporal super-resolution, which estimates the point spread function (blur kernel) from an image sequence [136138] or a single photograph [139] before deconvolution, or performs temporal super-resolution from a set of low rate videos [140,141], or even introduces hardware attachments [142].

\subsection{Extracting depth or shape}

The extraction of low level and middle level image cues is crucial for computer vision tasks. Computational photography can assist with the extraction in different ways.

1) Depth can be recovered from defocus analysis, because the depth of field is closely related to the distance. The typical approaches include introducing coded aperture patterns [46,143-145] or multiple apertures [114], computing from the image pairs captured using different aperture sizes [146-148,33]. Levin [149] compares the performances of different aperture codes in depth estimation and gives a mathematical analysis of the results using a geometrical optics model.

2) One can also compute the scene depth from the generalized illumination, e.g., casting a stripe pattern similar to structured light [62], using a multi-flash camera [60], or computing the depth from images taken under varying lighting conditions [67,150-152].

\section{Light field and its development}

Generally speaking, the objective of computational photography is to extend the low dimensional 2D subspace sampled by traditional photography to a higher level, and thus to acquire and display the visual information in more effective ways. The previous work in computational photography can be seen as an extension along a subset of the 7D plenoptic function [47], and in this, the most important work is the light field. In this section, we review the birth and subsequent development of studies of the light field and discuss their future directions.

\subsection{Plenoptic function and light field}

The definition of the plenoptic function originates from Gershun's idea of formulating light with a series of rays to describe its radiometric properties [153], including the directions of the rays $(\theta, \phi)$ at each point $\left(V_{x}, V_{y}, V_{z}\right)$, which compose the most important subspace of the popular 7D plenoptic function [1], which 

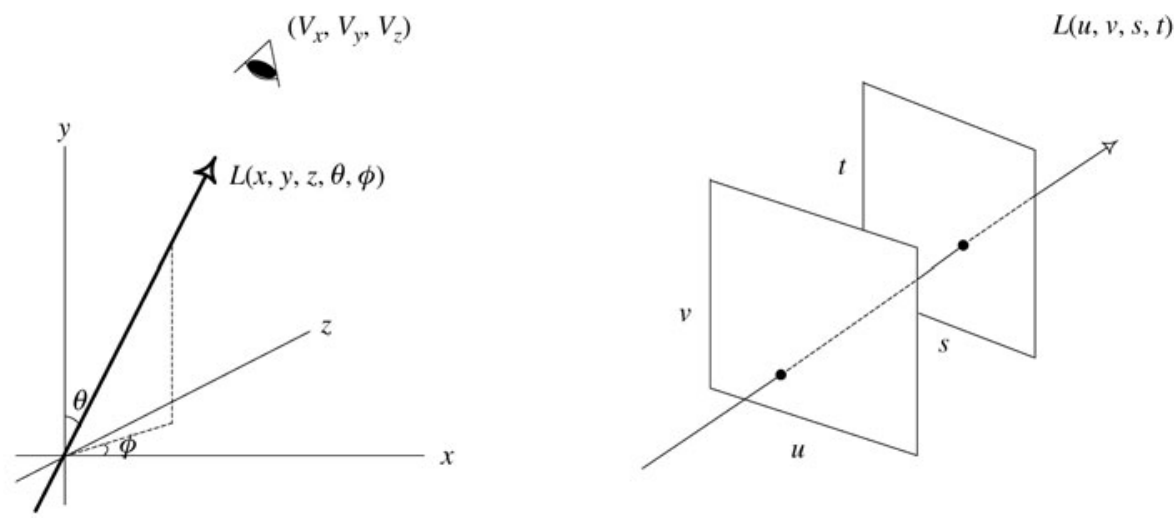

Figure 6 The plenoptic function and its bi-planar parameterization

was proposed by Adelson to formulate the dense array of light rays in the space, as shown in Figure 6(a). In the parameterization, the plenoptic function $P\left(\theta, \phi, \lambda, t, V_{x}, V_{y}, V_{z}\right)$ or $P\left(x, y, \lambda, t, V_{x}, V_{y}, V_{z}\right)$ includes 7 parameters: the spherical coordinates $P(\theta, \phi)$ or the Cartesian coordinates of the picture plane $P(x, y)$, the intensity variations with wavelength $\lambda$, the time dimension $t$, and the viewing position coordinates $V_{x}, V_{y}$ and $V_{z}$. This function can describe the visual information of any object, from any viewpoint, at any time constant, and within any wavelength range. Capturing the light field enables one to view the scenes from any point by simple re-sampling methods, while, with the geometry or reflection attributes unknown, thus helping with e.g. refocusing, extending the field of view, and real-time scene rendering, as reviewed in Section 3 .

Because the viewpoint is not an intrinsic property of the objects, the 7D plenoptic function is usually simplified to 5D. McMillan [154] views this 5D plenoptic function as a panoramic image at different locations. When considering only the convex hull of the object, the radiance along a ray remains constant from point to point along its length, so the plenoptic function can be further simplified to be a 4D, researchers in computer graphics call which light field [9] or Lumigraph [16]. In this paper, we refer to it as the light field.

Because the light field is highly complex, its parameterization is a nontrivial task. Researchers have tried several alternatives, among which the bi-planar form is the most popular one, as shown in Figure 6(b), and which was used in [9] and [16], among other works.

\subsection{Light field capture-plenoptic cameras}

The device recording the light field is called a light field camera or a plenoptic camera. The plenoptic camera's roots come from refs [68] and [155], and it usually requires a tradeoff between the sampled dimensions of the plenoptic function and some other costs, e.g., time, camera number, or spatial resolution. According to the costs for higher sampling dimensions, the available plenoptic cameras fall into three categories, as shown in Figure 7.

1) Sequential shots. In a sequential capture scheme, the light field is captured by taking snapshots of the subjects from different viewpoints sequentially, where each corresponds to one specific angular direction. The camera position can be determined in various ways, e.g. by mounting a controllable camera gantry [9], moving a hand-held camera along a specific surface [16], or inserting a pattern scroll or program controllable liquid crystal array (LCA) in front of the detector [4]. Figure 8(a) gives a representative sequential prototype, which is used in [9].

Two obvious shortcomings of sequential capture are: (i) it is inappropriate for use in photographing dynamic scenes; (ii) sequential capture is time consuming and needs elaborate camera control.

2) Simultaneous shots. To capture the light field of moving scenes, one can use a well-synchronized camera array to capture the objects from different viewpoints. Wilburn et al. [84] built a camera array which was tightly packed in a plane, and captured images from different views and positions. Using a similar planar system, Yang et al. [156] implemented two prototypes under a scalable camera array arch- 


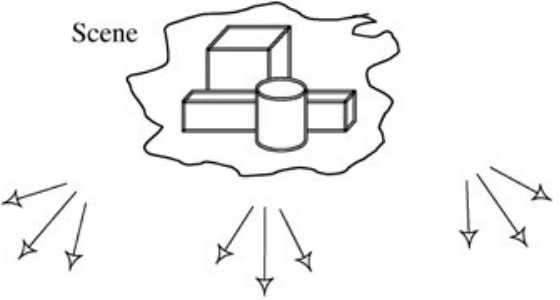

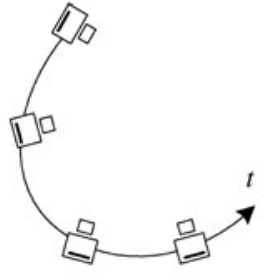

(a)

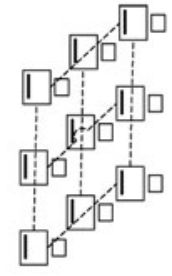

(b)

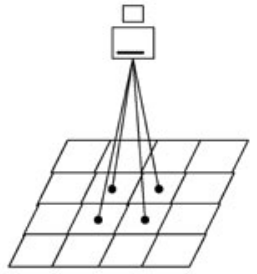

(c)

Figure 7 Three frequently adopted prototypes of plenoptic cameras. (a) Sequential shots; (b) simultaneous shots; (c) multiplexing capturing.

(a)

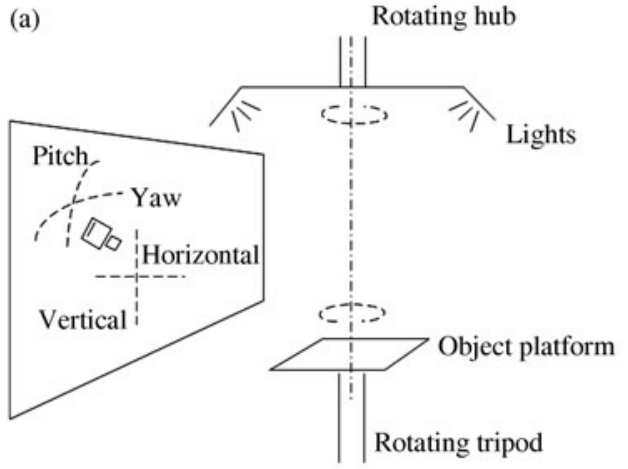

(b)

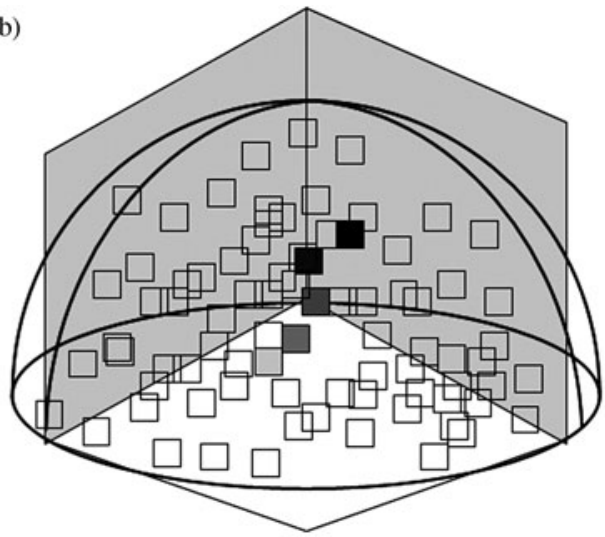

Figure 8 Two schemes for single lens plenoptic cameras adopted in [9] (a) and [16] (b).

itecture for real-time distributed light field capture. To capture light from all-round viewpoints and under varying illumination conditions, Einarsson et al. [157], Liu et al. [158] and Wu [159] all set up dome shaped light field capturing systems with cameras and LED lights under synchronized control.

Camera array based light field acquirement is advantageous compared to that based on single shots; however, the calibration (both geometric and color) of a large number of cameras is nontrivial, and the simultaneous control and large bandwidth are also complicated for large-scale system design.

3) Multiplexing. In signal processing, multiplexing is a technique of projecting high dimensional data onto a lower dimension, with a typical example being the widely-used Bayer pattern [160]. Ihrke et al.'s paper [76] puts much previous work in computational photography into this framework. In light field capture, multiplexing is also used to project multiple slices of the plenoptic function onto a $2 \mathrm{D}$ image.

Multiplexing can be implemented in the spatial domain, or Georgiev et al. [73] divided the plenoptic cameras into two subclasses, for the spatial design and the frequency design. The plenoptic cameras designed from the spatial viewpoint are usually implemented by inserting a microlens array $[12,2,155]$ or lens and prism combination [161] into the traditional camera for multiplexing the light rays from different directions. In contrast, the frequency domain modulation of the light field usually adopts configurations with one or more masks $[2,6,7,155,161,162]$ inserted near the aperture or sensor; this mask-based plenoptic camera method is often named the heterodyning method.

The captured images acquired via multiplexing are usually of lower resolution. Bishop et al. [119] performed light field superresolution by introducing priors (e.g. Lambertianity and texture statistics) or local structural patterns. 
Considering that a large number of light field cameras have been invented, Levin et al. [163] developed a platform for comparison of the different light field cameras under a Bayesian framework.

\subsection{Revolution of light field and its acquirement}

Because of its importance in new imaging mechanisms and the wide application foreground, researchers have been exploring new devices and schemes to capture the light field. In this subsection, we review the progress in this direction.

1) Static light field under consistent illumination. The static light field describes the static scenes from any direction and at any point. The continuous $4 \mathrm{D}$ static light field can be reconstructed from discrete sample points in the bi-planar parameter space, which can be acquired sequentially using a handheld camera, or simultaneously using a camera array.

Levoy et al. [9], and Gortler et al. [16] both adopted the sequential capture scheme, with the pose and position of a handheld camera under specific control. Levoy et al. [9] built a planar gantry for light field capture, as shown in Figure 8(a). They mounted the objects on a tripod and equipped the camera with pan and tilt motors for image capture from different viewpoints. The interface of Gortler et al.'s [16] capture system is a little different (see Figure 8(b)), as they 'painted' a half sphere surface surrounding the object using a real camera. For the camera control, they also built a special motion control platform to place the camera at positions and orientations coincident with the sample points in the parameter space.

With the light field captured, one can then render the expected images from any virtual viewpoint in a real-time manner. The reconstruction process can be carried out either pixel by pixel, ray by ray, or by introducing a texture mapping approach to improve the rendering efficiency.

Because the two light field models above capture the light field with a single camera, they cannot deal with varying illuminations and moving objects, and so the light field must be further developed for more complex cases. These situations are explained in the next two subsections.

2) Light field of cyclic motion under varying illuminations. To capture the light fields of moving scenes, Yang et al. [156] built a real-time distributed light field camera array system consisting of $8 \times 8$ video cameras, but it could not record and transmit whole scenes completely. Later, Tsuhan et al. [164] made some improvements and built a self-reconfigurable planar camera array, which could deal with non-rapid motion due to the slow speed of the camera motion and the elapsed computation time. For the light field under varying illumination, Yang et al. [50] built a dome shaped camera array system to capture both static and dynamic face images under different illumination conditions using moving light sources.

To extend the light fields in both the time and illumination dimensions, Wen et al. [107] used a similar dome system, which changed the illumination via a controlled diode composition. However, this system only targeted face sequences, and they later built an improved system for capture of the cyclic motion of humans [89], as shown in Figure 9. The system includes: (i) a treadmill on a turntable, where the subjects perform cyclic motions (e.g. walking, running); (ii) a vertical array of three high-speed cameras for motion capture; (iii) dome lights, evenly distributed on the top two-thirds of an $8 \mathrm{~m}$ geodesic sphere; and (iv) evenly spaced floor light units beneath the subject for simulation of the illumination from a Lambertian ground plane. Using such an apparatus, the authors recorded subjects with a sequence of illumination conditions repeating at $30 \mathrm{~Hz}$.

After acquiring the images, one can generate the light field under varying illumination conditions (referred to as a flow reflectance field) using the following steps: generating mattes of the tracking frame in each sequence, using a lighting basis registration which maps the images to the tracking frame, computing the flow between the vertical and horizontal viewpoints, and computing the shadows using a visual hull intersection. Images from a new viewpoint and under a new illumination can be synthesized based on the reconstructed light field by the following five steps: relighting, viewpoint interpolation, image warping, shadow simulation, and image compositing.

3) Light field of general 3D objects under varying illumination. Despite its considerable improvement over previous light field capture methods, the work discussed above was insufficient in several ways, e.g., the motion is limited to cyclic motions, the rendering results are sensitive to the accuracy of the optical 


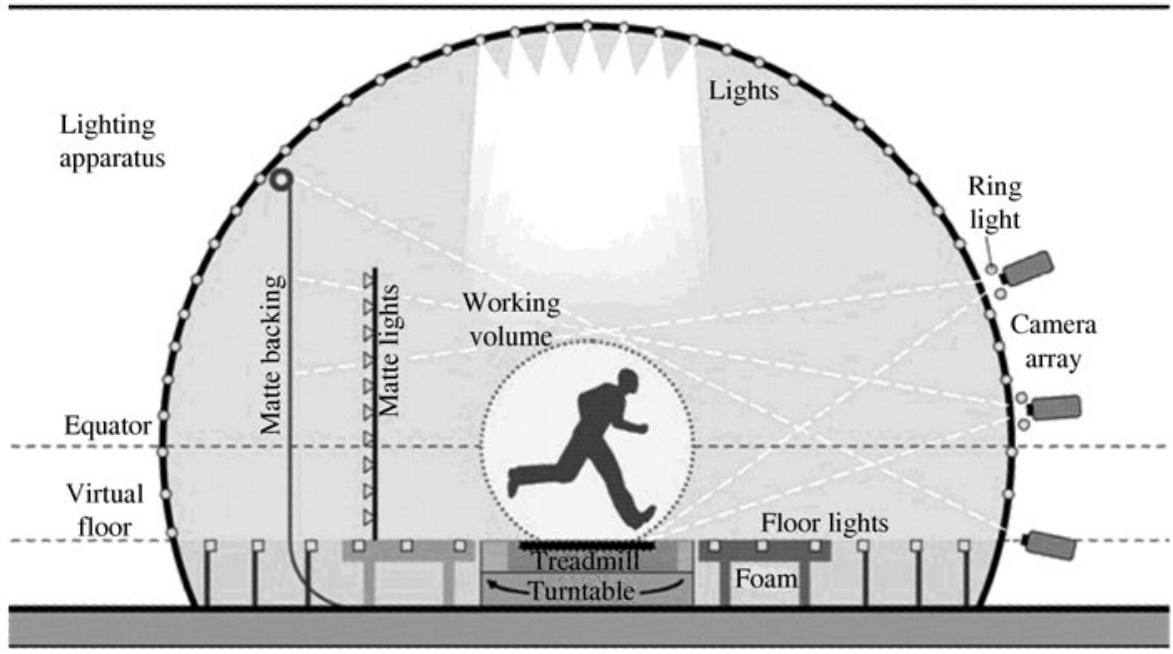

Figure 9 A dome shaped camera array used in [157] to capture light fields of cyclic motion under varying illumination conditions.

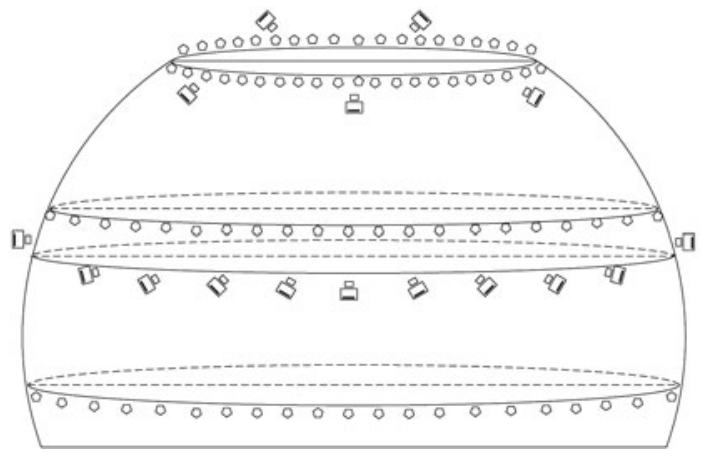

Figure 10 A camera array used in $[158,159]$ for capture of light fields of generic motion and under varying illumination conditions.

flow computation, and the system cannot be used to model a 3D geometry. Later, Liu [158] and Wu [159] extended the previous work to cover generic motion and 3D geometry capture.

Similar to the system in [67], the proposed capture system adopts a dome shaped architecture (see Figure 10), which includes three key modules: the mechanical module, which is a $6 \mathrm{~m}$ dome; the lighting system, which has 320 light sources evenly spaced on the hemisphere of the dome; and the capture system, which has 40 PointGrey Flea2 cameras arranged in a ring-shape on the dome, and a distributed synchronization controller module, in which each controller controls an LED subset and a camera subset and the different controllers are synchronized via the system clock. Using this capture system, Liu et al. proposed a continuous depth estimation method for generic 3D objects [165] and a point-cloud-based multi-view stereo algorithm for free-viewpoint dynamic scenes [166].

In Liu's work [158], the 3D reconstruction is implemented by using an improved multi-view stereo approach. Unlike the traditional stereo matching metric that is based on pixel intensity or color, Liu uses a surface normal weighted with a reflectance ratio for feature matching. Wu [159] later improved Liu's approach by introducing photometric constraints and surface consistency for more accurate reconstruction, and then further generalized the approach to include unknown illumination sources and obtain detailed reconstruction results.

Using this light field capture system, relighting was implemented by building geometric models for rendering of the changing viewpoints and by adopting sampling over densely captured images under varying illumination conditions. 


\subsection{The potential research directions and challenges}

Because of its feasibility, its intrinsic advantages and the wide application foreground, the research in light fields has received widespread attention. However, there are still a number of open issues to be resolved for light field capture.

1) Light field compression. The light field used to describe high-dimensional visual information is undoubtedly demanding on computer memory, especially for dynamic scenes. To compress the light field data, a number of methods have been tried, e.g., vector quantization $[9,167]$, temporal encoding [168], hierarchical prediction [169,170], model based texture mapping [171,172], wavelet transforms [173-175], and disparity compensated lifting $[176,177]$. However, with the increasing degrees of captured dimensions, light field compression remains a difficult issue.

2) Camera array design. Capture of high-dimensional light fields via a large camera array has become a popular approach in recent years, and the design of the camera array system requires a major effort, e.g. for the scalability of the system, synchronization control of the large numbers of cameras and light sources, and the color and geometric calibration of the cameras.

3) Capturing light fields of real world scenes. The current light field capture systems are constrained to specific subjects (e.g., face, human) or motions (e.g. cyclic motion), and the light sources are also mostly limited to simple LEDs, but photographed real-world scenes are often much more complex, and the light field capture of real scenes is a challenging but useful problem which deserves further investigation.

\section{The future of computational photography}

As an emerging and rapidly developing field, no one yet knows where computational photography may go from here and what may be achieved. In this section, we suggest some potential directions from the authors' perspective.

\subsection{Hardware improvements based on imaging theory}

The reviewed studies on computational photography mostly modify the imaging systems for better visual information capture, and are changing our views on photography. However, these works are all performed using traditional geometric optics; this limitation is not irrevocable. Taking a further step and extending traditional imaging theory to wave optics [178] and developing new imaging theory and imaging systems would be a revolutionary direction, which may enable many more new applications.

\subsection{Cognitive camera}

As mentioned previously, capturing all of the dimensions of the plenoptic function is the ultimate goal of computational photography, i.e., acquiring the full spectrum of scenes at any position, at any time instant, and from any direction. With the plenoptic visual information captured, we can then extract any task specific slices by using simple sampling computations, e.g. refocusing, relighting, or occlusion removal. We call this ideal capture device a 'cognitive camera'. However, developing a system that is able to acquire the whole plenoptic function is a challenging task. Besides the hardware design, the highdimensional data is memory-intensive, and requires a huge bandwidth. Therefore, capturing the effective subspaces of the plenoptic function based on the limited hardware resources is a central issue for plenoptic camera design. Many plenoptic function subspaces have been found in computational photography thus far. For a systematic review, we provide a framework for the various slices of the plenoptic function in Figure 11, which suggests some future research directions for computational photography.

\subsection{Higher intelligence: scene-specific capture mode}

Computational photography is a cross-disciplinary field, and thus developments in related fields, e.g. machine learning and computer vision, would undoubtedly have benefits for progress in computational 


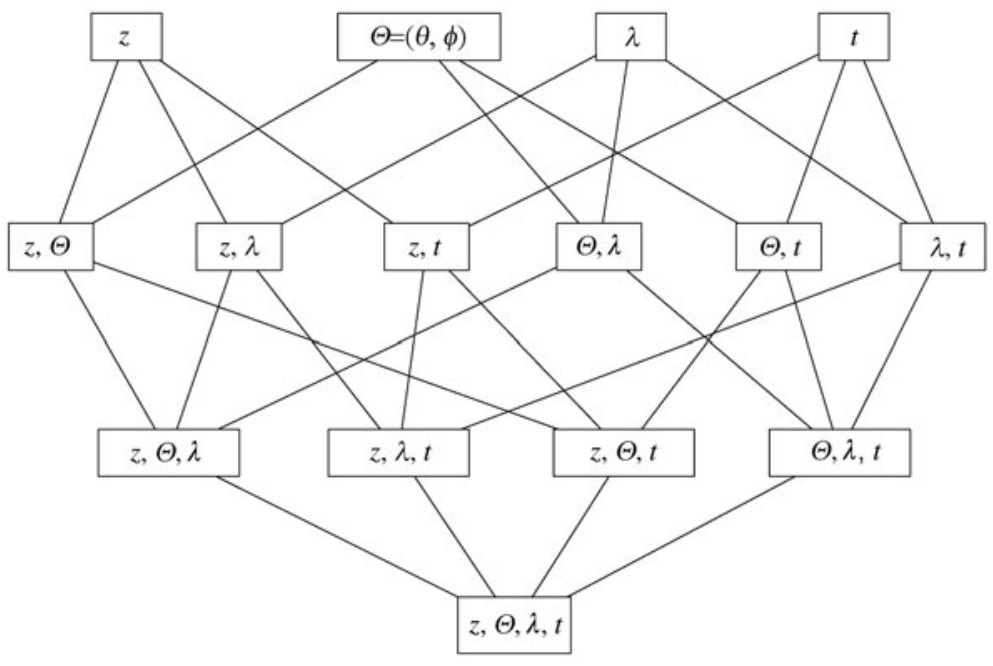

Figure 11 Different slices of 7D plenoptic function.

photography. Combining the progress in machine intelligence with imaging theory to design a taskspecific imaging mode is one direction for future photography applications, e.g. astronomical photography, medical imaging, and face photography.

\section{$6 \quad$ Summary and conclusions}

Computational photography is an emerging research field, which is developing rapidly thanks to the efforts of researchers from multiple disciplines. Because of the short history and the intrinsic cross-disciplinary characteristics of this field, there is neither a clear boundary nor a precise definition of computational photography.

Because computational photography is a multidisciplinary area, the research methods have some distinguishing features, e.g. closely combining hardware design and algorithm development, which call for considerable experimental work together with support of the basic theories in statistical learning, signal processing, and computer vision, providing a uniform platform for researchers from different fields, and requiring a uniform framework for field-specific theories. The development of computational photography will require cooperation and communication between many traditional scientific disciplines.

\section{Acknowledgements}

This work was supported by National Basic Research Project (Grant No.2010CB731800) and National Natural Science Foundation of China (Grant Nos. 61035002, 60972013, 61171119, 61120106003).

\section{References}

1 Adelson E H, Bergen J R. Computational Models of Visual Processing. Cambridge: MIT Press, 1991. 3-20

2 Adelson E H, Wang J Y A. Single lens stereo with a plenoptic camera. IEEE Trans Pattern Anal Mach Intell, 1992, 14: $99-106$

3 Zhou C, Nayar S K. What are good apertures for defocus deblurring? In: IEEE International Conference on Computational Photography (ICCP), Cluj-Napoca, 2009

4 Liang C K, Lin T H, Wong B Y, et al. Programmable aperture photography: Multiplexed light field acquisition. ACM Trans Graph, 2008, 27: 1-10

5 Dowski E R, Cathey W T. Extended depth of field through wave-front coding. Appl Optics, 1995, 34: 1859-1866

6 Veeraraghavan A, Raskar R, Agrawal A, et al. Dappled photography: Mask enhanced cameras for heterodyned light fields and coded aperture refocusing. ACM Trans Graph, 2007, 26: 69 
7 Agrawal A, Veeraraghavan A, Raskar R. Reinterpretable imager: Towards variable post-capture space, angle and time resolution in photography. In: Eurographics, Norrköping, 2010

8 Mohan A, Woo G, Hiura S, et al. Bokode: Imperceptible visual tags for camera based interaction from a distance. ACM Trans Graph, 2009, 28: 1-8

9 Levoy M, Hanrahan P. Light field rendering. In: International Conference on Computer Graphics and Interactive Techniques, New Orleans, 1996. 31-42

10 Agrawal A, Raskar R, Nayar S K, et al. Removing photography artifacts using gradient projection and flash-exposure sampling. ACM Trans Graph, 2005, 24: 828-835

11 Gallo O, Gelfand N, Chen W C, et al. Artifact-free high dynamic range imaging. In: IEEE International Conference on Computational Photography (ICCP), Cluj-Napoca, 2009

12 Ren N, Marc L, Mathieu B, et al. Light field photography with a hand-held plenoptic camera. Stanford University Computer Science Technical Report, 2005

13 Schechner Y Y, Nayar S K. Generalized mosaicing. In: IEEE International Conference on Computer Vision (ICCV), Vancouver, 2001. 17-24

14 Higo T, Matsushita Y, Joshi N, et al. A hand-held photometric stereo camera for 3-D modeling. In: IEEE International Conference on Computer Vision (ICCV), Kyoto, 2009

15 Levin A. Analyzing depth from coded aperture sets. In: European Conference on Computer Vision (ECCV), Crete, 2010

16 Gortler S J, Grzeszczuk R, Szeliski R, et al. The lumigraph. In: International Conference on Computer Graphics and Interactive Techniques (SIGGRAPH), New Orleans, 1996. 43-54

17 Narasimhan S G, Nayar S K. Enhancing resolution along multiple imaging dimensions using assorted pixels. IEEE Trans Pattern Anal Mach Intell, 2005, 27: 518-530

18 Nayar S K, Branzoi V, Boult T E. Programmable imaging using a digital micromirror array. In: IEEE International Conference on Computer Vision and Pattern Recognition (CVPR), Washington, 2004. 436-443

19 Krishnan D, Fergus R. Dark flash photography. ACM Trans Graph, 2009, 28: 1-11

20 Levin A, Zomet A, Peleg S, et al. Seamless image stitching in the gradient domain. In: European Conference on Computer Vision (ECCV), Prague, 2004. 377-389

21 Li W, Zhang J, Dai Q H. Exploring aligned complementary image pair for blind motion deblurring. In: IEEE International Conference on Computer Vision and Pattern Recognition (CVPR), Colorado Springs, 2011

22 Mohan A, Raskar R, Tumblin J. Agile spectrum imaging: Programmable wavelength modulation for cameras and projectors. Comput Graph Forum, 2008, 27: 709-717

23 Zhou C, Lin S, Nayar S K. Coded aperture pairs for depth from defocus. In: IEEE International Conference on Computer Vision (ICCV), Kyoto, 2009

24 Levin A, Freeman W T. 4D frequency analysis of computational cameras for depth of field extension. ACM Trans Graph, 2009, 28: 1-14

25 Green P, Sun W, Matusik W, et al. Multi-aperture photography. ACM Trans Graph, 2007, 26: 1-7

26 Zomet A, Nayar S K. Lensless imaging with a controllable aperture. In: IEEE International Conference on Computer Vision and Pattern Recognition (CVPR), New York, 2006. 339-346

27 Kuthirummal S, Nayar S K. Multiview radial catadioptric imaging for scene capture. ACM Trans Graph, 2006, 25: 916-923

28 Schechner Y Y, Nayar S K. Generalized mosaicing: Wide field of view multispectral imaging. IEEE Trans Pattern Anal Mach Intell, 2002, 24: 1334-1348

29 Nayar S K, Branzoi V, Boult T E. Programmable imaging: Towards a flexible camera. Int J Comput Vis, 2006, 70 : $7-22$

30 Horstmeyer R, Euliss G, Athale R, et al. Flexible multimodal camera using a light field architecture. In: IEEE International Conference on Computational Photography (ICCP), Cluj-Napoca, 2009

31 Raskar R, Agrawal A, Tumblin J. Coded exposure photography: motion deblurring using fluttered shutter. ACM Trans Graph 2006, 25: 795-804

32 Ng R. Fourier slice photography. ACM Trans Graph, 2005, 24: 735-744

33 Rajagopalan A N, Chaudhuri S. Optimal selection of camera parameters for recovery of depth from defocused images. In: IEEE International Conference on Computer Vision and Pattern Recognition (CVPR), San Juan, 1997. 219-224

34 Talvala E V, Adams A, Horowitz M, et al. Veiling glare in high dynamic range imaging. ACM Trans Graph, 2007, 26: $37-46$

35 Schechner Y Y, Nayar S K. Generalized mosaicing: Polarization panorama. IEEE Trans Pattern Anal Mach Intell 2005, 27: 631-636

36 Schechner Y Y, Nayar S K. Uncontrolled modulation imaging. In: IEEE International Conference on Computer Vision and Pattern Recognition (CVPR), Washington, 2004. II-197-II-204

37 Schechner Y Y, Nayar S K. Generalized mosaicing: High dynamic range in a wide field of view. Int J Comput Vis, 
2003, 53: $245-267$

38 Kuthirummal S, Nayar S K. Flexible mirror imaging. In: ICCV Workshop on Omnidirectional Vision, Camera Networks and Non-classical Cameras (OMNIVIS), Rio de Janeiro, 2007

39 Raskar R, Agrawal A, Wilson C, et al. Glare aware photography: 4D ray sampling for reducing glare effects of camera lenses. ACM Trans Graph, 2008, 27: 56-64

40 Nomura Y, Zhang L, Nayar S K. Scene collages and flexible camera arrays. In: Eurographics Symposium on Rendering, Grenoble, 2007. 127-138

41 Mohan A, Lanman D, Hiura S, et al. Image destabilization: Programmable defocus using lens and sensor motion. In: IEEE Conference on Computational Photography (ICCP), Cluj-Napoca, 2009

42 Nagahara H, Kuthirummal S, Zhou C, et al. Flexible depth of field photography. In: European Conference on Computer Vision (ECCV), Marseille, 2008

43 Levin A, Sand P, Cho T S, et al. Motion-invariant photography. ACM Trans Graph, 2008, 27: 1-9

44 Ben-Ezra M, Lin Z, Wilburn B. Penrose pixels: Super-resolution in the detector layout domain. In: IEEE International Conference on Computer Vision (ICCV), Rio de Janeiro, 2007

45 Tumblin J, Agrawal A, Raskar R. Why I want a gradient camera? In: International Conference on Computer Vision and Pattern Recognition (CVPR), San Diego, 2005. 103-110

46 Wetzstein G, Ihrke I, Heidrich W. Sensor saturation in fourier multiplexed imaging. In: IEEE International Conference on Computer Vision and Pattern Recognition (CVPR), San Francisco, 2010

47 Zhang C, Chen T. Light field sampling. Synth Lect Image Video Multimed Process, 2006, 2: 1-102

48 Cossairt O, Nayar S K, Ramamoorthi R. Light field transfer: Global illumination between real and synthetic objects. ACM Trans Graph, 2008, 27: 1-6

49 Park J, Lee M, Grossberg M D, et al. Multispectral imaging using multiplexed illumination. In: IEEE International Conference on Computer Vision (ICCV), Rio de Janeiro, 2007

50 Yang J C, Everett M, Buehler C, et al. A real-time distributed light field camera. In: Eurographics workshop on Rendering, Pisa, 2002. 77-85

51 Debevec P, Hawkins T, Tchou C, et al. Acquiring the reflectance field of a human face. In: International Conference on Computer Graphics and Interactive Techniques (SIGGRAPH), New Orleans, 2000. 145-156

52 Petschnigg G, Szeliski R, Agrawala M, et al. Digital photography with flash and no-flash image pairs. ACM Trans Graph, 2004, 23: 664-672

53 Schechner Y Y, Nayar S K, Belhumeur P N. Multiplexing for optimal lighting. IEEE Trans Pattern Anal Mach Intell, 2007, 29: 1339-1354

54 Eisemann E, Eisemann E, Durand F. Flash photography enhancement via intrinsic relighting. ACM Trans Graph, 2004, 23: 673-678

55 Feris R, Raskar R, Tan K, et al. Specular reflection reduction with multi-flash imaging. In: IEEE Brazilian Symposium on Computer Graphics and Image Processing (SIBGRAPI), Curitiba, 2004. 316-321

56 Dicarlo J M, Xiao F, Wandell B A. Illuminating illumination. In: Color Imaging Conference, Scottsdale, 2000

57 Crispell D, Lanman D, Sibley P G, et al. Beyond silhouettes: Surface reconstruction using multi-flash photography. In: 3rd International Symposium on 3D Data Processing, Visualization, Transmission, Chapel Hill, 2006. 405-412

58 Fattal R, Agrawala M, Rusinkiewicz S. Multiscale shape and detail enhancement from multi-light image collections. ACM Trans Graphics, 2007, 26: 51

59 Tan K, Feris R, Raskar R, et al. Harnessing real-world depth edges with multiflash imaging. IEEE Comput Graph Appl, 2005, 25: 32-38

60 Raskar R, Tan K H, Feris R, et al. Non-photorealistic camera: depth edge detection and stylized rendering using multi-flash imaging. ACM Trans Graph, 2004, 23: 679-688

61 Vaquero D A, Raskar R, Feris R S, et al. A projector-camera setup for geometry-invariant frequency demultiplexing. In: IEEE International Conference on Computer Vision and Pattern Recognition (CVPR), Miami, 2009. 2082-2089

62 Zhang L, Nayar S K. Projection defocus analysis for scene capture and image display. ACM Trans Graph, 2006, 25: 907-915

63 Nayar S K, Krishnan G. Visual chatter in the real world. In: Proceedings of Eurographics Symposium on Rendering, Nicosia, 2006. 11-16

64 Nayar S K, Krishnan G, Grossberg M D, et al. Fast separation of direct and global components of a scene using high frequency illumination. ACM Trans Graph, 2006, 25: 935-944

65 Malzbender T, Gelb D, Wolters H. Polynomial texture maps. In: International Conference on Computer Graphics and Interactive Techniques (SIGGRAPH), Los Angeles, 2001. 519-528

66 Masselus V, Peers P, Dutre P, et al. Relighting with 4D incident light fields. ACM Trans Graph, 2003, 22: 613-620

67 Wenger A, Gardner A, Tchou C, et al. Performance relighting and reflectance transformation with time multiplexed illumination. ACM Trans Graph, 2005, 24: 756-764

68 Liao M, Wang L, Yang R G. Light falloff stereo. In: IEEE Conference on Computer Vision and Pattern Recognition 
(CVPR), Minneapolis, 2007

69 Matusik W, Pfister H, Ngan A, et al. Image-based 3D photography using opacity hulls. ACM Trans Graph, 2002, 21: $427-437$

70 Anrys F, Dutre P. Image based lighting design. In: International Conference on Visualization, Imaging, Image Processing (VIIP), Marbella, 2004

71 Koppal S J, Narasimhan S G. Time-constrained photography. In: IEEE International Conference on Computer Vision (ICCV), Kyoto, 2009. 333-340

72 Veeraraghavan A, Agrawal A, Raskar R, et al. Non-refractive modulators for encoding and capturing scene appearance and depth. In: IEEE International Conference on Computer Vision and Pattern Recognition (CVPR), Anchorage, 2008

73 Georgiev T, Intwala C, Babacan S, et al. Unified frequency domain analysis of lightfield cameras. In: Europeon Conference on Computer Vision (ECCV), Marseille, 2008

74 Alleysson D, Ssstrunk S, Herau J. Linear demosaicing inspired by the human visual system. IEEE Trans Image Process, 2005, 14: 439-449

75 Lanman D, Raskar R, Agrawal A, et al. Shield fields: Modeling and capturing 3D occluders. ACM Trans Graph, 2008, 27: $1-10$

76 Ihrke I, Wetzstein G, Heidrich W. A theory of plenoptic multiplexing. In: IEEE International Conference on Computer Vision and Pattern Recognition (CVPR), San Francisco, 2010

77 Adams A, Talvala E, Park S H, et al. The Frankencamera: An experimental platform for computational photography. ACM Trans Graph, 2010, 19: 1-12

78 Taguchi Y, Agrawal A, Ramalingam S, et al. Axial light field for curved mirrors: Reflect your perspective, widen your view. In: IEEE Conference on Computer Vision and Pattern Recognition (CVPR), San Francisco, 2010

79 Agarwala A, Agarwala M, Cohen M, et al. Photographing long scenes with multi-viewpoint panoramas. ACM Trans Graph, 2006, 25: 853-861

80 Agarwala A, Dontcheva M, Agrawala M, et al. Interactive digital photomontage. ACM Trans Graph, 2004, 23: 294-302

81 Davis J. Mosaics of scenes with moving objects. In: IEEE International Conference on Computer Vision and Pattern Recognition (CVPR), Santa Barbara, 1998. 354-360

82 Szeliski R, Shum H Y. Creating full view panoramic image mosaics and environment maps. In: International Conference on Computer Graphics and Interactive Techniques (SIGGRAPH), Los Angeles, 1997. 251-258

83 Uyttendaele M. Eliminating ghosting and exposure artifacts in image mosaics. In: International Conference on Computer Vision and Pattern Recognition (CVPR), Kauai, 2001. 509-516

84 Wilburn B, Joshi N, Vaish V, et al. High performance imaging using large camera arrays. ACM Trans Graph, 2005, 24: $765-776$

85 Agarwala A, Zheng C, Pal C, et al. Panoramic video textures. ACM Trans Graph, 2005, 24: 821-827

86 Hasinoff S W, Durand F, Freeman W T. Noise-optimal capture for high dynamic range photography. In: IEEE International Conference on Computer Vision and Pattern Recognition (CVPR), San Francisco, 2010

87 Mcguire M, Matusik W, Pfister H, et al. Optical splitting trees for high-precision monocular imaging. IEEE Comput Graph Appl, 2007, 27: 32-42

88 Burt P J, Kolczynski R J. Enhanced image capture through fusion. In: IEEE International Conference on Computer Vision (ICCV), Berlin, 1993. 173-182

89 Debevec $\mathrm{P}$, Wenger A, Tchou C, et al. A lighting reproduction approach to live-action compositing. ACM Trans Graph, 2002, 21: 547-556

90 DiCarlo J, Wandell B. Rendering high dynamic range images. In: The International Society for Optical Engineering (SPIE), San Diego, 2000. 392-401

91 Madden B C. Extended intensity range imaging. University of Pennsylvania, GRASP Laboratory Technical Report, 1993

92 Mann S, Picard R W. On being 'undigital' with digital cameras: Extending dynamic range by combining differently exposed pictures. In: Proceedings of IS and Ts 48th Annual Conference, Washington, 1995. 442-448

$93 \mathrm{Lu} \mathrm{P} \mathrm{Y,} \mathrm{Huang} \mathrm{T} \mathrm{H}$, Wu M S, et al. High dynamic range image reconstruction from hand-held cameras. In: IEEE International Conference on Computer Vision and Pattern Recognition (CVPR), Miami, 2009

94 Aggarwal M, Ahuja N. Split aperture imaging for high dynamic range. Int J Comput Vis, 2004, 58: 7-17

95 Wang H C, Raskar R, Ahuja N. High dynamic range video using split aperture camera. In: IEEE 6th Workshop on Omnidirectional Vision, Camera Networks and Non-classical Cameras (OMNIVIS, in conjunction with ICCV), Beijing, 2005

96 Morimura A. Imaging method for a wide dynamic range and an imaging device for a wide dynamic range. US Patent, 5455621,1995

97 Nayar S K, Mitsunaga T. High dynamic range imaging: Spatially varying pixel exposures. In: IEEE International 
Conference on Computer Vision and Pattern Recognition (CVPR), Hilton Head Island, 2000. 472-479

98 Bennett E P, McMillan L. Video enhancement using per-pixel virtual exposures. ACM Trans Graph, 2005, 24: 845-852

99 Street R A. High dynamic range segmented pixel sensor array. US Patent, 5789737, 1998

100 Nayar S K, Narasimhan S G. Assorted pixels: Multisampled imaging with structural models. In: European Conference on Computer Vision (ECCV), Copenhagen, 2002. 636-652

101 Mitsunaga T, Nayar S K. Radiometric self calibration. In: IEEE International Conference on Computer Vision and Pattern Recognition (CVPR), Ft. Collins, 1999. 374-380

102 Knight T F. Design of an integrated optical sensor with on-chip preprocessing. PhD thesis. Massachusetts: Massachusetts Institute of Technology, 1983

103 Brajovic V, Kanade T. A sorting image sensor: An example of massively parallel intensity-to-time processing for low-latency computational sensors. In: IEEE International Conference on Robotics and Automation, Minneapolis, 1996. 1638-1643

104 Scheffer D, Kavadias S, Dierickx B, et al. A logarithmic response CMOS image sensor with on-chip calibration. IEEE J Solid State Chem, 2000, 35: 1146-1152

105 Decker S J, McGrath R D, Brehmer K, et al. A 256x256 CMOS imaging array with wide dynamic range pixels and column-parallel digital output. In: IEEE International Conference on Solid-State Circuits, San Francisco, 1998. $176-177$

106 Handy R J. High dynamic range CCD detector imager. US Patent, 4623928, 1986

107 Wen D D. High dynamic range charge coupled device. US Patent, 4873561, 1989

108 Hamazaki M. Non-linear photosite response in CCD imagers. US Patent, RE34802, 1994

109 Hamazaki M. Driving method for solid-state image pickup device. US Patent, 5990952, 1999

110 Cossairt O, Zhou C, Nayar S K. Diffusion coding photography for extended depth of field. ACM Trans Graph, 2010, 29: $1-10$

111 Hasinoff S W, Kutulakos K N. Light-efficient photography. In: European Conference on Computer Vision (ECCV), Marseille, 2008

112 Telleen J, Sullivan A, Yee J, et al. Synthetic shutter speed imaging. In: European Association for Computer Graphics, Prague, 2007. 591-598

113 Hasinoff S W, Kutulakos K N, Durand F, et al. Time-constrained photography. In: IEEE International Conference on Computer Vision (ICCV), Kyoto, 2009. 333-340

114 Guichard F, Nguyen H P, Tessieres R, et al. Extended depth-of-field (eDof) using sharpness transport across color channels. In: The International Society for Optical Engineering (SPIE), Chernivtsi, 2009

115 Isaksen A, McMillan L, Gortler S J. Dynamically reparameterized light fields. In: International Conference on Computer Graphics and Interactive Techniques (SIGGRAPH), New Orleans, 2000. 279-306

116 Horowitz M, Ng R, Adams A. Light field microscopy. ACM Trans Graph, 2006, 25: 924-934

117 Du H, Tong X, Cao X, et al. A prism-based system for multispectral video acquisition. In: IEEE International Conference on Computer Vision (ICCV), Kyoto, 2009. 175-182

118 Cao X, Du H, Tong X, et al. A prism-mask system for multispectral video acquisition. IEEE Trans Pattern Anal Mach Intell, 2011, 33: 2423-2435

119 Bishop T E, Zanetti S, Favaro P. Light field superresolution. In: IEEE International Conference on Computational Photography (ICCP), Cluj-Napoca, 2009

120 Landolt O, Mitros A, Koch C. Visual sensor with resolution enhancement by mechanical vibrations. In: International Conference Advanced Research in VLSI, Salt Lake City, 2001. 233-239

121 Wang S, Heidrich W. The design of an inexpensive very high resolution scan camera system. Comput Graph Forum, 2004, 23: 441-450

122 Ben-Ezra M. High resolution large format tile-scan camera: Design, calibration, extended depth of field. In: IEEE International Conference on Computational Photography (ICCP), Cambridge MA, 2010

123 Cossairt O S, Miau D, Nayar S K. Gigapixel computational imaging. In: IEEE International Conference on Computational Photography (ICCP), Pittsburg, 2011

124 Kirmani A, Hutchison T, Davis J, et al. Looking around the corner using transient imaging. In: IEEE International Conference on Computer Vision (ICCV), Kyoto, 2009. 159-166

125 Wilburn B, Joshi N, Vaish V, et al. High performance imaging using large camera arrays. ACM Trans Graph, 2005, 24: $765-776$

126 Tai Y W, Du H, Brown M S, et al. Correction of spatially varying image and video motion blur using a hybrid camera. IEEE Trans Pattern Anal Mach Intell, 2010, 32: 1012-1028

127 Agrawal A, Raskar R. Resolving objects at higher resolution from a single motion-blurred image. In: IEEE International Conference on Computer Vision and Pattern Recognition (CVPR), Minneapolis, 2007

128 Agrawal A, Raskar R. Optimal single image capture for motion deblurring. In: IEEE International Conference on Computer Vision and Pattern Recognition (CVPR), Miami, 2009. 2560-2567 
129 Agrawal A, Xu Y. Coded exposure deblurring: Optimized codes for PSF estimation and invertibility. In: IEEE International Conference on Computer Vision and Pattern Recognition (CVPR), Miami, 2009. 2066-2073

130 Agrawal A, Xu Y, Raskar R. Invertible motion blur in video. ACM Trans Graph, 2009, 28: 95

131 Veeraraghavan A, Reddy D, Raskar R. Coded strobing photography: Compressive sensing of high-speed periodic events. IEEE Trans Pattern Anal Mach Intell, 2011, 33: 671-686

132 Agrawal A, Gupta M, Veeraraghavan A. Optimal coded sampling for temporal super-resolution. In: IEEE Conference on Computer Vision and Pattern Recognition (CVPR), San Francisco, 2010. 599-606

133 McCloskey S. Velocity-dependent shutter sequences for motion deblurring. In: Europeon Conference on Computer Vision (ECCV), Crete, 2010

134 Ezra M B, Nayar S K. Motion-based motion deblurring. IEEE Trans Pattern Anal Mach Intell, 2004, 26: 689-698

135 Ezra M B, Zomet A, Nayar S K. Jitter camera: High resolution video from a low resolution detector. In: IEEE Conference on Computer Vision and Pattern Recognition (CVPR), Washington, 2004. II-135-II-142

136 Ezra M B, Nayar S K. Motion deblurring using hybrid imaging. In: IEEE Conference on Computer Vision and Pattern Recognition (CVPR), Madison, 2003. I-657-I-664

137 Ezra M B, Nayar S K. Motion-based motion deblurring. IEEE Trans Pattern Anal Mach Intell, 2004, 26: 689-698

138 Bascle B, Blake A, Zisserman A. Motion deblurring and super-resolution from an image sequence. In: European Conference on Computer Vision (ECCV), Freiburg, 1996. 573-582

139 Fergus R, Singh B, Hertzmann A, et al. Removing camera shake from a single photograph. ACM Trans Graph, 2006, 25: $787-794$

140 Shechtman E, Caspi Y, Irani M. Increasing space-time resolution in video. In: European Conference on Computer Vision (ECCV), Copenhagen, 2002. 753-768

141 Shechtman E, Caspi Y, Irani M. Space-time super-resolution. IEEE Trans Pattern Anal Mach Intell, 2005, 27: 531-545

142 Joshi N, Kang S B, Zitnick C. L, et al. Image deblurring using inertial measurement sensors. ACM Trans Graph, 2010, 29: 1-9

143 Levin A, Fergus R, Durand F, et al. Image and depth from a conventional camera with a coded aperture. ACM Trans Graph, 1996, 26: 1-9

144 Nayar S K, Nakagawa Y. Shape from focus. IEEE Trans Pattern Anal Mach Intell, 1994, 16: 824-831

145 Vaish V, Szeliski R, Zitnick C L, et al. Reconstructing occluded surfaces using synthetic apertures: Stereo, focus and robust measures. In: IEEE International Conference on Computer Vision and Pattern Recognition (CVPR), New York, 2006. 2331-2338

146 Favaro P, Soatto S. A geometric approach to shape from defocus. IEEE Trans Pattern Anal Mach Intell, 2005, 27: 406-417

147 Watanabe M, Nayar S K. Rational filters for passive depth from defocus. Int J Comput Vis, 1998, 27: 203-225

148 Nayar S K, Watanabe M, Noguchi M. Real-time focus range sensor. IEEE Trans Pattern Anal Mach Intell, 1996, 18: 1186-1198

149 Levin A. Analyzing depth from coded aperture sets. In: European Conference on Computer Vision (ECCV), Crete, 2010

150 Zickler T, Belhumeur P N, Kriegman D J. Helmholtz stereopsis: Exploiting reciprocity for surface reconstruction. Int J Comput Vis, 2002, 49: 869-884

151 Akers D, Losasso F, Rick J. Conveying shape and features with image-based relighting. In: IEEE Visualization (VIS), Seattle, 2003. 349-354

152 Basri R, Jacobs D. Photometric stereo with general, unknown lighting. In: IEEE International Conference on Computer Vision and Pattern Recognition (CVPR), Kauai, 2001. II-374-II-381

153 Gershun A. The light field. J Math Phys, 1936, 18: 51-151

154 McMillan L. Plenoptic modeling: An image-based rendering system. In: International Conference on Computer Graphics and Interactive Techniques (SIGGRAPH), Los Angeles, 1995. 39-46

155 Javidi B, Frauel Y. Three-dimensional object visualization and recognition based on computational integral imaging. Opt Eng, 1999, 38: 1072-1077

156 Yang J C, Everett M, Buehler C, et al. A real-time distributed light field camera. In: Eurographics workshop on Rendering, Pisa, 2002. 77-86

157 Einarsson P, Chabert C F, Jones A, et al. Relighting human locomotion with flowed reflectance fields. In: Eurographics Symposium on Rendering, Nicosia, 2006. 183-194

158 Liu Y. Key Technologies in Light field. PhD thesis. Beijing: Tsinghua University, 2009

$159 \mathrm{Wu}$ C L. Key technologies on multi-view reconstruction under multiple illuminations. Master's thesis. Beijing: Tsinghua University, 2010

160 Bayer B E. Color imaging array. US Patent, 3971065, 1976

161 Georgeiv T, Zheng K C, Curless B, et al. Spatio-angular resolution tradeoff in integral photography. In: Eurographics Symposium on Rendering, Nicosia, 2006. 263-272 
162 Lumsdaine A, Georgiev T. The focused plenoptic camera. In: IEEE International Conference on Computational Photography (ICCP), Cluj-Napoca, 2009

163 Levin A, Freeman W T, Durand F. Understanding camera trade-offs through a Bayesian analysis of light field projections. In: European Conference on Computer Vision (ECCV), Marseille, 2008

164 Tsuhan C Z, Zhang C, Chen T. A self-reconfigurable camera array. In: Eurographics Symposium on Rendering, Sweden, 2004. 243-253

165 Liu Y, Cao X, Dai Q H, et al. Continuous depth estimation for multi-view stereo (CMVS). In: IEEE International Conference on Computer Vision and Pattern Recognition (CVPR), Miami, 2009. 2121-2128

166 Liu Y, Dai Q, Xu W. A point cloud based multi-view stereo algorithm for free-viewpoint video. IEEE Trans Vis Comput Graph, 2010, 16: 407-418

167 Tong X, Gray M G. Interactive view synthesis from compressed light fields. In: International Conference on ImageProcessing (ICIP), Catalonia, 2003. 85-88

168 Matusik W, Pfister H. 3DTV: A scalable system for real-time acquisition, transmission, autostereoscopic display of dynamic scenes. ACM Trans Graph, 2004, 23: 814-824

169 Marcus M, Girod B. Data compression for light field rendering. IEEE Trans Circuits Syst Video Technol, 2000, 10: 338-343

170 Magnor M, Girod B. Hierarchical coding of light fields with disparity maps. In: International Conference on Image Processing (ICIP), Kobe, 1999. 334-338

171 Sebe I O, Ramanathan P, Girod B. Multi-view geometry estimation for light field compression. In: Vision, Modelling and Visualization, Erlangen, 2002. 265-272

172 Magnor M, Ramanathan P, Girod B. Multi-view coding for image-based rendering using 3-D scene geometry. IEEE Trans Circuits Syst Video Technol, 2003, 13: 1092-1106

173 Magnor M, Endmann A, Girod B. Progressive compression and rendering of light fields. In: International Workshop on Vision, Modeling, Visualization (VMV), Stuttgart, 2000. 199-203

174 Xu D, Dai Q H, Xu W L. Data compression of light field using wavelet packet. In: IEEE International Conference on Multimedia and Expo (ICME), Taipei, 2004

$175 \mathrm{Xu}$ D, Dai Q H, Xu W L. Light field compression based on prediction propagating and wavelet packet. In: IEEE International Conference on Image Processing (ICIP), Singapore, 2004. 3515-3518

176 Chang C L, Zhu X Q, Ramanathan P, et al. Light field compression using disparity-compensated lifting and shape adaptation. IEEE Trans Image Process, 2006, 15: 793-806

177 Girod B, Chang C L, Ramanathan P, et al. Light field compression using disparity-compensated lifting. In: IEEE International Conference on Acoustics, Speech, Signal Processing (ICASSP), Hong Kong, 2003. 761-764

178 Zhang Z Y, Levoy M. Wigner distributions and how they relate to the light field. In: IEEE International Conference on Computational Photography (ICCP), Cluj-Napoca, 2009 